\title{
Transient Heat Conduction through a Substrate of Brine-Spongy Ice
}

S.R. Dehghani ${ }^{1}$, G.F. Naterer, Y.S. Muzychka \begin{abstract}
Department of Mechanical Engineering, Faculty of Engineering and Applied Science,
\end{abstract} Memorial University of Newfoundland, St. John’s, NL, A1B 3X5, Canada

\section{Abstract}

This paper develops a new predictive model of transient heat transfer in brine-spongy ice. It analyzes the thermal behavior and discusses the thermal response of brine-spongy ice to heat input. The model is based on the global characteristics of a mixture of ice and brine. The analytical model uses phase change characteristics of brine and ice in equilibrium states. The model involves a nonlinear partial differential equation and a number of equations of state for ice, brine, and brinespongy ice. Salinity, initial temperature, and a constant temperature for one side of the ice block, are primary variables in the studies. Samples are also tested experimentally with transient conditions and their temperature responses are captured by a thermal camera. The numerical results are closely aligned with the measured temperatures. The experiments confirm the analytical model and numerical solutions. This paper establishes new understanding of trapped brine when absorbing and releasing thermal energy through brine-spongy ice. The observations indicate a new type of ice structure with considerable differences in thermal response to transient heat transfer as compared with regular ice.

Keywords: Brine-Spongy Ice, Transient Conduction, Latent Heat, Salinity, Phase Change

\footnotetext{
${ }^{1}$ Corresponding author, Postdoctoral Fellow, Phone: +1 (709) 771-6216, Email: srdehghani@mun.ca
} 


\section{Nomenclature}

\begin{tabular}{|c|c|c|}
\hline Variable & Unit & Definition \\
\hline$C_{b}$ & $\mathrm{~J} /(\mathrm{Kg} . \mathrm{K})$ & Specific heat capacity of brine \\
\hline$C_{e}$ & $\mathrm{~J} /(\mathrm{Kg} . \mathrm{K})$ & Equivalent specific heat capacity of brine-spongy ice \\
\hline$C_{i}$ & $\mathrm{~J} /(\mathrm{Kg} . \mathrm{K})$ & Specific heat capacity of ice \\
\hline$C_{s}$ & $\mathrm{~J} /(\mathrm{Kg} . \mathrm{K})$ & Specific heat capacity of brine-spongy ice \\
\hline$k_{b}$ & $\mathrm{~W} /(\mathrm{m} \cdot \mathrm{K})$ & Thermal conductivity of brine \\
\hline$k_{i}$ & $\mathrm{~W} /(\mathrm{m} . \mathrm{K})$ & Thermal conductivity of ice \\
\hline$k_{s}$ & $\mathrm{~W} /(\mathrm{m} . \mathrm{K})$ & Thermal conductivity of brine-spongy ice \\
\hline$L$ & $\mathrm{~m}$ & Ice sample length \\
\hline$L_{H b}$ & $\mathrm{~J} / \mathrm{Kg}$ & Latent heat of fusion of brine \\
\hline$Q_{i}$ & $\mathrm{~J} / \mathrm{s}$ & Input heat \\
\hline$Q_{o}$ & $\mathrm{~J} / \mathrm{s}$ & Output heat \\
\hline$Q_{g}^{\prime \prime \prime}$ & $\mathrm{J} / \mathrm{m}^{3}$ & Volumetric heat generation \\
\hline$\dot{Q}_{g}^{\prime \prime \prime}$ & $\mathrm{J} /\left(\mathrm{m}^{3} \cdot \mathrm{s}\right)$ & Rate of volumetric heat generation \\
\hline$S$ & $\%$ & Overall salinity \\
\hline$S_{b}$ & $\%$ & Salinity of brine \\
\hline$T$ & ${ }^{\circ} \mathrm{C}$ & Temperature \\
\hline$T_{S}$ & ${ }^{\circ} \mathrm{C}$ & Contact temperature \\
\hline$t$ & $s$ & Time \\
\hline$t_{0}$ & $s$ & Initial time \\
\hline$V_{b}$ & $\mathrm{~m}^{3}$ & Volume of brine in the element of brine-spongy ice \\
\hline$V_{F b}$ & --- & Volume fraction of brine \\
\hline
\end{tabular}




\begin{tabular}{|c|c|c|}
\hline$V_{i}$ & $\mathrm{~m}^{3}$ & Volume of ice in the element of brine-spongy ice \\
\hline$\alpha_{c b}$ & --- & Coefficient of the equation of specific heat capacity of brine \\
\hline$\alpha_{c i}$ & --- & Coefficient of the equation of specific heat capacity of ice \\
\hline$\alpha_{k b}$ & --- & Coefficient of the equation of thermal conductivity of brine \\
\hline$\alpha_{k i}$ & --- & Coefficient of the equation of thermal conductivity of ice \\
\hline$\alpha_{L H b}$ & --- & Coefficient of the equation of latent heat of fusion of brine \\
\hline$\alpha_{s b}$ & --- & Coefficient of the equation of salinity of brine \\
\hline$\alpha_{\rho b}$ & --- & Coefficient of the equation of density of brine \\
\hline$\alpha_{\rho i}$ & --- & Coefficient of the equation of density of ice \\
\hline$V_{s}$ & $\mathrm{~m}^{3}$ & Volume of the element of brine-spongy ice \\
\hline$d Q_{g}^{\prime \prime \prime}$ & $\mathrm{J} / \mathrm{m}^{3}$ & Variation of volumetric heat generation \\
\hline$d t$ & s & Time interval \\
\hline$d V_{F b}$ & --- & Variation of volume fraction of brine \\
\hline$d V_{i}$ & $\mathrm{~m}^{3}$ & Variation of volume of ice \\
\hline$\rho_{b}$ & $\mathrm{Kg} / \mathrm{m}^{3}$ & Density of brine \\
\hline$\rho_{e}$ & $\mathrm{Kg} / \mathrm{m}^{3}$ & Equivalent density of brine spongy ice \\
\hline$\rho_{i}$ & $\mathrm{Kg} / \mathrm{m}^{3}$ & Density of ice \\
\hline$\rho_{S}$ & $\mathrm{Kg} / \mathrm{m}^{3}$ & Density of brine spongy ice \\
\hline
\end{tabular}

\section{1. Introduction}

26 Icing of marine vessels, offshore structures, aircraft, overhead power lines, and wind turbines has

27 been a serious challenge for many years (Panov, 1978; Zakrzewski and Lozowski, 1988; Lozowski 28 et al., 2000). Ice accretion on marine vessels can cause many difficulties and hazardous situations 
29 (Ryerson, 2011). The type and amount of accumulated ice on marine vessels greatly depend on

30 the seawater temperature, wind speed, wind temperature, marine object geometry and velocity, 31 seawater salinity and other known and unknown parameters (Kulyakhtin and Tsarau, 2014; Gates 32 et al. 1986; Lozowski et al., 2000).

Although various situations of icing phenomena lead to the creation of different types and 34 structures of ice, it is known that some brine is always trapped between the solid ice structures during the icing phenomena and consequently makes a unique blend of ice and brine (Gates et al., 1986; Makkonen, 1987; Makkonen, 1990). This type of ice, in this paper, is called brine-spongy 37 ice. When a portion of salty water starts to freeze, it rejects the dissolved salt, and as a result, pure ice in the vicinity of the brine is created (Weeks and Ackley, 1982; Shakeel, 1987). In many cases, 39 the pure ice is developed by growth of dendritic ice (Glicksman, 2011). This type of ice formation increases the likelihood of trapping brine between the dendrites (Shakeel, 1987; Glicksman, 2011).

41 During the icing, a small amount of brine is trapped between the pure ice structures. This mixture 42 of ice and trapped brine is created layer by layer, and in fact the brine-spongy-ice accretion mechanism holds the main role in icing marine vessels (Panov, 1978; Gates et al., 1986; Makkonen 44 1990).

The state of equilibrium between brine and ice depends on the salinity of the brine pockets 46 and the local temperature (Weeks and Ackley, 1982; Shakeel, 1987). Increasing or decreasing the 47 salinity of the brine pockets causes melting and icing respectively (Weeks, 2010). In addition, 48 varying the temperature moves the state of equilibrium toward melting or icing (Shakeel, 1987; 49 Weeks, 2010). As spongy ice loses heat and becomes colder, the volume of trapped brine decreases 50 and that of pure ice is increased (Shakeel, 1987). Due to the decrease in temperature, the salt is 51 rejected into the brine pockets and the salinity of the brine pockets increases (Weeks and Ackley, 
52 1982; Shakeel, 1987; Weeks, 2010). Decreasing salinity and temperature create a new state of

53 equilibrium for ice and brine in a brine-spongy ice medium (Shakeel, 1987; Blackmore and

54 Lozowski, 1998).

55 The brine-spongy ice usually experiences various contact temperatures and heat transfer 56 conditions (Panov, 1978; Gates et al., 1986; Lozowski et al., 1996.). Due to the trapped brine inside

57 the ice cavity, the transient thermal behavior of brine-spongy ice is not solely heat conduction;

58 phase change plays a significant role (Blackmore and Lozowski, 1998). The freezing temperature

59 of trapped brine depends on its local salinity (Shakeel, 1987; Weeks and Ackley, 1982). The

60 freezing point of pure water is close to zero degrees Celsius and it decreases as the salinity

61 increases. The other thermo-physical properties of brine pockets are functions of salinity and 62 temperature (Shakeel, 1987).

The transient thermal behavior of brine-spongy ice has not been well understood in the

64 literature. The trapped brine in the ice structure can cause significant changes in the response of 65 this medium to transient heat transfer (Shakeel, 1987). In this study, analytical models of heat 66 transfer for brine-spongy ice are developed including with a numerical scheme, which is based on 67 a finite difference method. An experimental test rig is also used to conduct the relevant experiments 68 for verification of the analytical model and numerical results.

\section{Heat Transfer Formulation}

71 The thermal behavior of brine-spongy ice is strongly dependent on its overall salinity and local

72 temperature (Shakeel, 1987; Weeks and Ackley, 1982). In brine-spongy ice, the higher overall 73 salinity and temperature result in higher amounts of trapped brine ice and consequently the thermal 
74 behavior closer to the brine. On the other hand, a lower temperature and lower overall salinity lead 75 to conditions similar to a solid-ice-salt condition (Makkonen, 1987; Macklin and Ryan, 1962). The variable freezing point and heat of fusion of trapped brine in brine-spongy ice create a 77 complicated ice-brine region that can affect the thermal behavior of a block of brine-spongy ice 78 (Blackmore et al., 2002; Blackmore and Lozowski, 2003; Lozowski et al., 2005). Figure 1 shows 79 a schematic view of brine-spongy ice. The brine pockets are confined by pure ice. Pure ice and brine pockets are in a local equilibrium state. The salinity of a brine pocket is directly dependent

81 on its local temperature. This means by increasing the temperature, some pure ice is melted and 82 the salinity of brine pockets is decreased, and vice versa. Steady state heat transfer in pure ice and brine-spongy ice transfers the sensible heat energy 84 across the region (Weeks and Ackley, 1982). In the case of transient heat transfer, the behaviors are different. The heat transfer in pure ice is again limited to transferring sensible heat, but for brine-spongy ice, the heat of fusion has an important role. In brine-spongy ice, thermal energy 87 cannot pass through the media exclusively as sensible heat (Shakeel, 1987; Weeks and Ackley, 1982). The passage of heat affects the local temperature of brine-spongy ice and consequently, 89 some small amounts of ice melt and join the brine pockets and vice versa.

Figure 2 illustrates a brine-spongy ice medium which is affected by a sudden flux of heat

91 from the left side for a defined period. Once heating is started, thermal energy moves from the 92 higher temperature toward the lower temperature region, which is the right side of the medium. 93 This causes an increase in the temperature of the ice and brine pockets in its path. Increasing the 94 temperature of brine pockets and ice creates a new state of equilibrium and consequently, part of 95 the ice is melted and joined to its neighbor brine pockets. In this circumstance, the volume of brine 96 pockets is increased and the volume of ice is decreased. As a result, the volume fraction of brine, 
97 which is the ratio of volume of brine pockets to ice volume, is increased (Shakeel, 1987; Weeks 98 and Ackley, 1982). After a short time, heat affects part of this medium, which is named the heated

zone. In this zone the brine pockets have become larger than those of the unheated zone. The thermal energy cannot pass through any section of brine-spongy ice without melting or solidifying of a small portion of the ice or brine. This means some part of the transient thermal energy is absorbed in heating the local medium including ice and brine pockets, and some part is absorbed in melting a small amount of ice, while the rest can pass through, and vice versa. Figure 3 shows differences in the energy passage and storage in a segment of brine-spongy ice and pure ice which are affected by the heat input.

The governing equations for heat transfer in brine-spongy ice media can be derived by considering a small element of brine-spongy ice. Figure 4 shows a small element of brine-spongy ice at an initial time of $t=t_{0}$ and after a short time of $d t, t=t_{0}+d t$. The element contains a brine pocket which is confined by pure ice. After a short time, and due to entering heat, some ice is melted and it joins the brine pocket. Therefore the volume of the brine pocket is increased and the volume of pure ice is decreased. The salinity of the brine pocket is decreased because of the addition of new melted ice to it. The brine and ice absorb sensible heat and as a result their temperatures are higher. The heat of fusion for melting ice is the other part of the heat exchange. The rest of the heat input passes through the element and exits from the other sides.

The rate of volumetric heat generation in the element can be calculated from the density of ice, the melted ice volume, the heat of fusion of brine, and the volume of the element. The generated heat which is a result of phase change depends on the amount of phase change. Eq. (1) shows the rate of volumetric heat generation in a short period of time.

$$
\frac{d Q_{g}^{\prime \prime \prime}}{d t}=\frac{-\rho_{i} d V_{i} L_{H b}}{V_{S} d t}
$$


121

122

123

124

125

126

127

128

129

130

131

132

133

134

135

136

137

138

139

140

141

the rate of volumetric heat generation in an element of brine-spongy ice can be obtained.

$$
\begin{aligned}
& \frac{d Q_{g}^{\prime \prime \prime}}{d t}=\rho_{i} L_{H b} \frac{d V_{F b}}{d t} \\
& \dot{Q}_{g}^{\prime \prime \prime}=\rho_{i} L_{H b} \frac{\partial V_{F b}}{\partial t}
\end{aligned}
$$

Eq. (3) shows the amount of volumetric heat generation in an element of brine-spongy ice due to the time variation of volume fraction of brine in the element. By knowing the overall salinity of the spongy ice, the volume fraction of brine can be calculated for each temperature. Eq. (4) is an empirical relation that calculates the volume fraction for any temperature and overall salinity (Weeks and Ackley, 1982; Weeks 2010).

$$
V_{F b}=10^{-3} S\left(-\frac{49.185}{T}+0.532\right)
$$

By using Eq. (3) and Eq. (4), a new relation for heat generation, which is dependent on the overall salinity, temperature, and time variation of temperature, is obtained.

The governing equation for transient heat transfer (Ozisik, 1993) with heat generation is

$$
\rho_{s} C_{s} \frac{\partial T}{\partial t}=\vec{\nabla} \cdot\left(k_{s} \vec{\nabla} T\right)+\dot{Q}_{g}^{\prime \prime \prime}
$$

Eq. (5), which is a heat conduction equation for a medium with a moving heat source, can be used as a governing equation for heat transfer in brine-spongy ice. The interface of phase change acts as a moving heat source. Melting absorbs the latent heat of fusion, and icing releases the latent heat of fusion. The volumetric rate of heat generation of the phase change is calculated by the derivative of $V_{F b}$. Using this equation and replacing $\dot{Q}_{g}^{\prime \prime \prime}$ in Eq. (5), the governing equation of heat transfer in a brine-spongy ice is obtained as follows:

$$
\begin{gathered}
\rho_{e} C_{e}=\left(\rho_{s} C_{S}-\frac{\rho_{i} L_{H b} S 49.185 \times 10^{-3}}{T^{2}}\right) \\
\rho_{e} C_{e} \frac{\partial T}{\partial t}=\vec{\nabla} \cdot\left(k_{s} \vec{\nabla} T\right)
\end{gathered}
$$


142 This partial differential equation is dependent on overall salinity and thermal properties of brine 143 pockets, ice, and brine-spongy ice. Table 1 shows relations which describe the thermal properties 144 of ice, brine, and brine-spongy ice (Weeks and Ackley, 1982; Weeks 2010; Cox and Weeks 1975). 145 Eq. (4), Eq. (7), and the equations of properties in Table 1 yield 13 equations and 13 146 unknowns leading to a set of nonlinear equations. The unknowns are temperature, brine 147 conductivity, brine heat capacity, brine salinity, brine density, brine heat of fusion, ice density, ice 148 thermal conductivity, ice heat capacity, spongy ice conductivity, spongy ice density, spongy ice 149 heat capacity, and volume fraction of brine. Properties of spongy ice are considered as weighted 150 properties of ice and brine. The properties in Table 1 are empirical relations of previous 151 investigators (Weeks and Ackley, 1982; Weeks 2010; Cox and Weeks 1975). These are dependent 152 on the coefficients which are obtained experimentally. Table 2 includes the coefficients in the 153 equations of Table 1 (Weeks and Ackley, 1982; Weeks 2010; Cox and Weeks, 1975). With an 154 initial condition, which is the initial distribution of temperature in the brine-spongy ice, the 155 boundary conditions, and also the overall salinity of brine-spongy ice, this set of 13 equations and 15613 unknowns can be solved. The results will predict the thermal behavior of brine-spongy ice at 157 each moment of time.

\section{Experimental Investigation}

160 A cold plate, for which the coolant is supplied from a cold bath containing a mixture of water and 161 glycol, maintains a wide range of low temperatures from zero to $-25^{\circ} \mathrm{C}$. The cooling capacity of 162 the cold plate is enough to maintain the temperature at a set point. A cold bath, which is filled with 163 a glycol-water mixture, supplies the chilled liquid for the cold plate. A thermal camera, which is 164 calibrated for subzero temperatures with an accuracy of $\pm 0.1{ }^{\circ} \mathrm{C}$ measures the temperatures of 
target points and areas. The experiments are conducted with a cold plate which is adjusted to a set point equal to the initial temperature of a block of brine-spongy ice. The size of the samples and the magnitude of the heat transfer between the samples and the cold plate are such that the cold plate can accurately maintain a set temperature during the experiments. The cold plate keeps one side of the ice samples at a chosen temperature between $-5^{\circ} \mathrm{C}$ and $-20^{\circ} \mathrm{C}$. Figure 5 illustrates the experimental setup and the most significant elements involved in the experiments.

Various samples with different salinities are created and kept in a freezer, equipped with a temperature controller, to reach the desired temperature. A uniform temperature is established throughout the ice. The experimental tests are conducted by using salinities between zero and 65\%, a fixed initial temperature, which is $-20^{\circ} \mathrm{C}$, and a fixed temperature for the cold plate which is $-5^{\circ} \mathrm{C}$. The main measured parameter is the variation of the end temperature of the ice block over time. The variation of temperature over time can confirm the role of brine pockets in the thermal behavior of brine-spongy ice.

The samples are made by mixing water and appropriate amounts of salt. The saline samples are placed in small containers and placed in a freezer with the target initial temperatures. Mixing and shaking of the containers during freezing aids in the creation of the homogeneous brine-spongy ice. The samples are kept at $-20^{\circ} \mathrm{C}$ for about a day. They are then brought out of the containers and kept for another day at $-20^{\circ} \mathrm{C}$. At this point they are ready for the experiments. The cold plate maintains the contact temperatures and the samples are placed directly on the cold plate. The thermal camera starts to capture the temperatures of the end side of the ice blocks.

\section{Results and Discussion}


187 The governing equations of brine-spongy ice are solved for obtaining the transient heat transfer in 188 brine-spongy ice in different situations. A one-dimensional case is considered. Figure 6 shows a 189 Cartesian one-dimensional problem of transient heat transfer in brine-spongy ice. The right- side 190 of the medium experiences a thermally isolated condition, and the left- side has a constant191 temperature condition, $T_{s}$. The overall salinity and initial temperature, which is a constant 192 temperature throughout the ice, are known. The sizes of brine pockets are not significant in 193 comparison to the length scale of brine-spongy ice. Homogeneous small pockets of trapped brine 194 make an almost uniform blend of ice and brine medium.

The governing equations for different cases of brine-spongy ice media with various initial and boundary conditions are solved and the results are compared and discussed. Overall salinity 197 can change the state of equilibrium significantly; increasing the volume of trapped brine, which 198 occurs because of increasing overall salinity, is one of the expected results of examining the brine199 spongy ice media.

As discussed, the main outstanding feature of brine-spongy ice appears in transient 201 situations. In these circumstances, the contribution of brine pockets in generation, absorption, and 202 transfer of heat is significant. An investigated parameter is the thermal response of brine-spongy 203 ice to time. In this case, the initial temperature of brine-spongy ice is considered $-20^{\circ} \mathrm{C}$, and the 204 left side of the ice block, $T_{s}$, is kept at a constant temperature of $-5^{\circ} \mathrm{C}$. The ice thickness is 20 $205 \mathrm{~mm}$. Figure 7 shows the thermal response of brine-spongy ice to a transient problem during the 206 time of $t=0 \mathrm{~s}$ to $\mathrm{t}=300 \mathrm{~s}$. The warmer side of the ice block, the left side, affects the spongy ice and starts to transfer 208 heat inside the ice. The heat penetrates the spongy ice from the left side of the block, and a portion 209 of ice which is closest to the left side, is affected by the heat immediately. Figure 7 shows the 
210 profiles of temperature across the spongy ice at various moments of time. As penetration of the

211 heat into the spongy ice is increased, the spongy ice becomes warmer. The temperature variation

212 in the block of spongy ice shows that after one second only $15 \%$ of the medium is affected by the

213 heat input. The remaining $85 \%$ is unheated at this time. The penetration of heat is increased by

214 increasing the time; at $\mathrm{t}=15 \mathrm{~s}$ the heated zone is about $50 \%$ of the medium. After about $60 \mathrm{~s}$ the

215 whole medium is heated. At this time the right-side temperature is approximately equal to the

216 initial temperature of the medium. At $t=180 \mathrm{~s}$ and $\mathrm{t}=300 \mathrm{~s}$, the penetrated heat increases the

217 right-side temperatures to $-17^{\circ} \mathrm{C}$ and $-15^{\circ} \mathrm{C}$ respectively.

218 As the brine-spongy ice becomes warmer, the state of equilibrium between the brine 219 pockets and the ice shifts to melt more ice. The melted ice is added to the brine pockets and as a 220 result the salinity of the brine pockets is decreased. Figure 8 shows that after one second, the 221 salinity of brine pockets in the $15 \%$ heated zone is decreased. The minimum salinity of brine 222 pockets in the whole region, which occurs at the left side, is about $80 \%$, which is considerably less 223 than the initial salinity of brine pockets, which is about 225\%. As time passes, the heat penetrates 224 more and the heated zone is increased, the melted ice is increased, and consequently the salinity 225 of the brine pockets decreases. The salinity of the brine pockets on the right side will be about $226 \quad 190 \%$ after $300 \mathrm{~s}$.

227 Melting the ice and joining the melted ice with the brine pockets can increase the volume 228 of the brine pockets and reduce the volume of ice and consequently, the volume fraction of brine 229 is increased. Figure 9 shows that as time increases, the volume fraction of brine is increased. The 230 maximum volume fraction of brine, which occurs on the left side, is about 0.67 . This means $67 \%$ 231 of the volume of brine-spongy ice at this point is occupied by brine pockets. The rest of the volume 
232 is occupied by the ice. The initial volume fraction of the brine is about $19 \%$, and after a short time 233 and in accordance with the amount of heat absorption, it can be increased to higher values.

235 of brine-spongy ice. Increasing the overall salinity changes the state of equilibrium toward freezing

236 the ice at a lower temperature. Figure 10 shows the thermal response of the brine-spongy ice to 237 various salinities. The numerical solutions are conducted in situations of an initial condition of $238-20^{\circ} \mathrm{C}$, and a boundary condition of $-5^{\circ} \mathrm{C}$. In addition, the overall salinity can vary between zero 239 and $65 \%$. The results are given for $\mathrm{t}=300 \mathrm{~s}$.

As Fig. 10 shows that the pure ice is greatly affected by the boundary condition, $\mathrm{T}=-5^{\circ} \mathrm{C}$.

241 After $300 \mathrm{~s}$ the temperature of the pure ice is increased from $-20^{\circ} \mathrm{C}$ to a range of $-7^{\circ} \mathrm{C}$ to $-5^{\circ} \mathrm{C}$.

242 The responses of high-saline cases, however, are completely different. For the high-salinity ice, 243 the temperature is between $-15^{\circ} \mathrm{C}$ and $-5^{\circ} \mathrm{C}$. This means the temperature across the high-salinity 244 ice is affected by the boundary less than that of the pure ice. This occurs because of the contribution 245 of the brine pockets in the process of heat storage inside the brine-spongy ice. Some heat is stored 246 in the brine pockets by melting the ice, and as a result the sensible heat cannot increase the 247 temperature of the medium significantly.

248 As the model predicts, increasing the overall salinity increases the salinity of brine pockets.

249 Figure 11 confirms that the salinity of the brine pockets for an overall salinity of $65 \%$ can be up to $250190 \%$. In this case the salinity of the brine pockets for an overall salinity of $5 \%$ cannot be more 251 than $120 \%$. The salinity of brine pockets which is closer to the left side is less than that of the right 252 side. The warmer side melts more ice and therefore decreases the salinity of its vicinity brine 253 pockets. 
The other interesting fact is the volume fraction of brine. In Fig. 12, by increasing the 255 overall salinity, the volume fraction of the brine increases. For the left side, which is affected by 256 the heat input, this variation is more considerable. The brine volume fraction of the pure ice is 257 zero, as expected. The highest volume fraction of brine occurs in the highest overall salinity case, 258 which is the case of an overall salinity of $65 \%$. In this case, the volume fraction of brine can be up 259 to 0.67 . This means that about $67 \%$ of the brine-spongy ice is occupied by the brine pockets in the 260 left-side.

The heat input from the left-side is caused by the high temperature of the left-side boundary 262 condition, which is a contact temperature. This temperature is high in comparison to the initial 263 temperature of the medium. The thermal responses for a block of brine-spongy ice, which is 264 affected by various contact temperatures, has a length of $20 \mathrm{~mm}$, an overall salinity of 65\%, and 265 an initial temperature of $-20^{\circ} \mathrm{C}$. Figure 13 shows the thermal responses of this medium for 266 different contact temperatures from $-5^{\circ} \mathrm{C}$ to $-20^{\circ} \mathrm{C}$.

267 For a contact temperature of $-20^{\circ} \mathrm{C}$, the temperature of the ice and the contact temperature 268 are the same and they are in a thermal equilibrium. Therefore, no heat transfer or changes in the 269 ice are expected. As differences between the contact temperature and the initial temperature of the 270 ice are increased, the rate of heat input increases and consequently the changes in the brine-spongy 271 ice appear. The most affected case is that of the contact temperature of $-5^{\circ} \mathrm{C}$. In this case, the 272 right- side temperature, the insulated side, reaches $-15^{\circ} \mathrm{C}$, while the right- side temperature in the 273 case of $-15^{\circ} \mathrm{C}$ is about $-18^{\circ} \mathrm{C}$.

As with other cases, the greatest change in salinity of the brine pockets is the case with the 275 maximum heat input. For the case of a contact temperature of $-5^{\circ} \mathrm{C}$, the salinity of brine pockets 276 reduces to $80 \%$ and the volume fraction of the brine goes up to 0.67 . These changes are less for 
277 the cases with lower contact temperatures. Figure 14 shows the variation of salinity of brine 278 pockets in various contact temperatures.

The insulated side is also affected by the variation of right- side temperatures. After $300 \mathrm{~s}$, 280 the minimum salinity of the brine pockets occurs for the highest contact temperature. It is about 281 180\% in the right- side. Higher contact temperature melts more ice and consequently increases the 282 volume fraction of the brine pockets in the warmer regions. Figure 15 shows the effects of 283 increasing of volume fraction of brine in various contact temperatures. The right-side, which is not 284 affected by heating after 300 s, has less variation of volume fraction.

285 The initial temperature of brine-spongy ice is the other important factor in the thermal 286 behavior of this ice. A lower initial temperature can cause trapped brine with a higher level of 287 salinity and a lower volume fraction. As the initial temperature increases, the brine-spongy ice will 288 contain a lower level of salinity of brine pockets and a higher volume fraction. Figure 16 shows 289 the thermal response of brine-spongy ice at some different initial temperatures. The solutions are 290 presented for an overall salinity of $65 \%$ and the results are given for $t=300$ s. The initial temperature 291 of $-20^{\circ} \mathrm{C}$ causes the lowest temperature in the right-hand side. The higher initial temperatures will 292 result in a higher temperature in the right-hand side.

The salinity of brine pockets, which is a function of temperature, is affected by initial 294 temperatures. The highest salinity corresponds to the lowest initial temperature. Because the right295 side is cooler than the left-side, the salinity of brine pockets in the right-hand side is more than that 296 of the left-side. Figure 17 shows the variation of salinity of brine pockets at various initial 297 temperatures.

The changes in the salinity of the brine pockets and the volume fraction of the brine are 299 related to each other. They experience higher variations with an initial temperature of $-20^{\circ} \mathrm{C}$. The 
other initial temperatures create some changes in the temperature, salinity of brine pockets, and

301 volume fraction of brine as well. As a common result, the higher initial temperatures cause less

302 changes in the temperature, salinity of brine pockets, and brine volume fraction. Figure 18 shows

303 the variation of volume fraction of brine at various initial temperatures. The lowest volume fraction

304 occurs for the coldest points, which are the right-side. The volume fraction in the case of $-20^{\circ} \mathrm{C}$

305 remains the minimum volume fraction in the whole medium.

The experimental tests are performed for various overall salinities. The overall salinities

307 are varied from zero, for fresh water, to 65\%. Figure 19 shows a comparison between the measured 308 temperatures and the numerical results. The numerical results for fresh water are in good 309 agreement with the experimental results. The measured temperatures are a little lower than the 310 numerical results. This occurs because of small trapped air bubbles in the ice. As the overall 311 salinity increases, the brine-spongy ice is less affected by the heat input. The error is lowest for 312 the highest overall salinity. In this case the error is about 5\%. The measured temperatures are again 313 lower than the numerical results. The errors may occur because of the thermo-physical properties 314 of brine and ice, trapped air bubbles, numerical solutions and also temperature measurements by 315 the thermal camera. The experimental results provide useful validation of the numerical solution. 316 The maximum error is about 5\%.

\section{5. Conclusions}

319 A new predictive model of transient heat transfer in brine-spongy ice was developed. This model 320 was obtained by assuming a homogeneous distribution of small brine pockets in brine-spongy ice.

321 The properties of pure ice, brine pockets and a mixture of ice and brine are used to address the 322 properties of brine-spongy ice. The contribution of melting and solidification of ice and brine 
323 pockets leads to a sensible-latent heat transfer process through the brine-spongy ice. A numerical

324 method is used to solve the governing equations. The numerical solutions show that brine-spongy

325 ice is less impacted by the heat input than pure ice. The temperature of the end point of a sample

326 of brine-spongy ice varies by about $5^{\circ} \mathrm{C}$, while in the same situation the end point of pure ice varies

327 by about $13^{\circ} \mathrm{C}$. Brine pockets absorb a portion of the heat input by melting the surrounding ice.

328 Melted ice increases the volume fraction of brine and consequently the salinity of brine pockets is

329 decreased. Also, an experimental setup using a calibrated thermal camera measures the end point

330 temperature of the block of brine-spongy ice. The experimental results provide useful validation

331 of the predictive model. The measured temperatures are less than $5 \%$ lower than the numerical

332 results.

333

334 Acknowledgments

335 The authors gratefully acknowledge the financial support of Statoil (Norway), MITACS, and 336 Petroleum Research Newfoundland and Labrador (PRNL) for this research.

\section{References}

339 Blackmore, R.Z., Lozowski, E.P., 1998. A theoretical spongy spray icing model with surficial 340 structure. Atmospheric Research, 49(4), 267-288.

341 Blackmore, R.Z., Lozowski, E.P., 2003. Spongy icing modelling: Progress and prospects. The 342 Thirteenth International Offshore and Polar Engineering Conference, 25-30 May, Honolulu, 343 Hawaii, USA.

344 Blackmore, R.Z., Makkonen, L., Lozowski E.P., 2002. A new model of spongy icing from first 345 principles. Journal of Geophysical Research, 107(D21), AAC 9-1-AAC 9-15. 
346 Cox, C.F.N., Weeks, W.F., 1975. Brine drainage and initial salt entrapment in sodium chloride

347 ice. U.S. Army Cold Regions Research and Engineering Laboratory, Research Report 345.

348 Gates, E.M., Narten, R., Lozowski, E.P., Makkonen, L., 1986. Marine icing and spongy ice. IAHR

349 Ice Symposium, 153-163.

350 Glicksman, M.E., 2011. Mechanism of dendritic branching. Metallurgical and Materials

351 Transactions A, 43(2), 391-404.

352 Kulyakhtin, A., Tsarau, A., 2014. A time-dependent model of marine icing with application of 353 computational fluid dynamics. Cold Regions Science and Technology, 104-105, 33-44.

354 Lozowski, E.P., Blackmore, R.Z., Forest, T.W., Shi, J., 1996. Spongy icing in the marine 355 environment. OMAE, Volume IV, 55-61.

356 Lozowski, E.P., Oleskiw, M., Blackmore, R.Z., Karev, A., Kolar, L., Farzaneh, M., 2005. Spongy

357 Icing Revisited: Measurements of Ice Accretion Liquid Fraction in Two Icing Tunnels. 43rd AIAA

358 Aerospace Sciences Meeting and Exhibit, Aerospace Sciences Meetings.

359 Lozowski, E.P., Szilder, K., Makkonen, L., 2000. Computer simulation of marine ice accretion. 360 Royal Society, 358, 2811-2845.

361 Macklin, W.C., Ryan, B.F., 1962. On the formation of spongy ice. Quarterly Journal of the Royal 362 Meteorological Society, 88(378), 548-549.

363 Makkonen, L., 1987. Salinity and growth rate of ice formed by sea spray. Cold Region Science 364 and Technology, 14, 163-171.

365 Makkonen, L., 1990. The origin of spongy ice. IAHR Ice Symposium, 1022-1030.

366 Ozisik, M.N., 1993. Heat Conduction. John Wiley \& sons, Inc.

367 Panov, V.V., 1978. Icing of Ships. Polar Geography, 2 (3), 166-186. 
368 Ryerson, C.C., 2011. Ice protection of offshore platforms. Cold Regions Science and Technology, $36965(1), 97-110$.

370 Shakeel, T., 1987. Dynamics of spontaneous pattern formation in dendritic ice crystal growth. PhD 371 Thesis, State University of New York.

372 Zakrzewski, W.P., Lozowski, E.P., 1988. Estimating the extent of the spraying zone on a sea373 going ship. Ocean Engineering, 15(5), 413-429.

374 Weeks, W.F., 2010. On sea ice. University of Alaska Press.

375 Weeks, W.F., Ackley, S.F., 1982. The growth, structure, and properties of sea ice. CRREL 376 Monograph 82-1.

377 378 379 380 381 382 383 384 385 386 


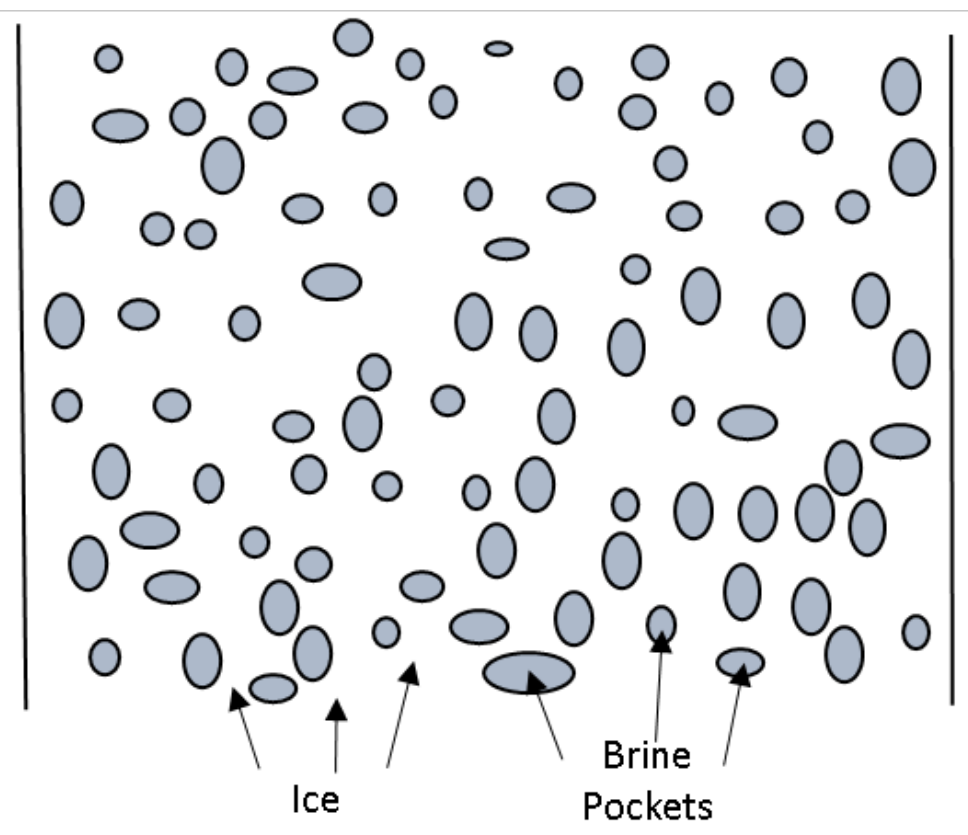

Fig. 1. Schematic of brine-spongy ice

389

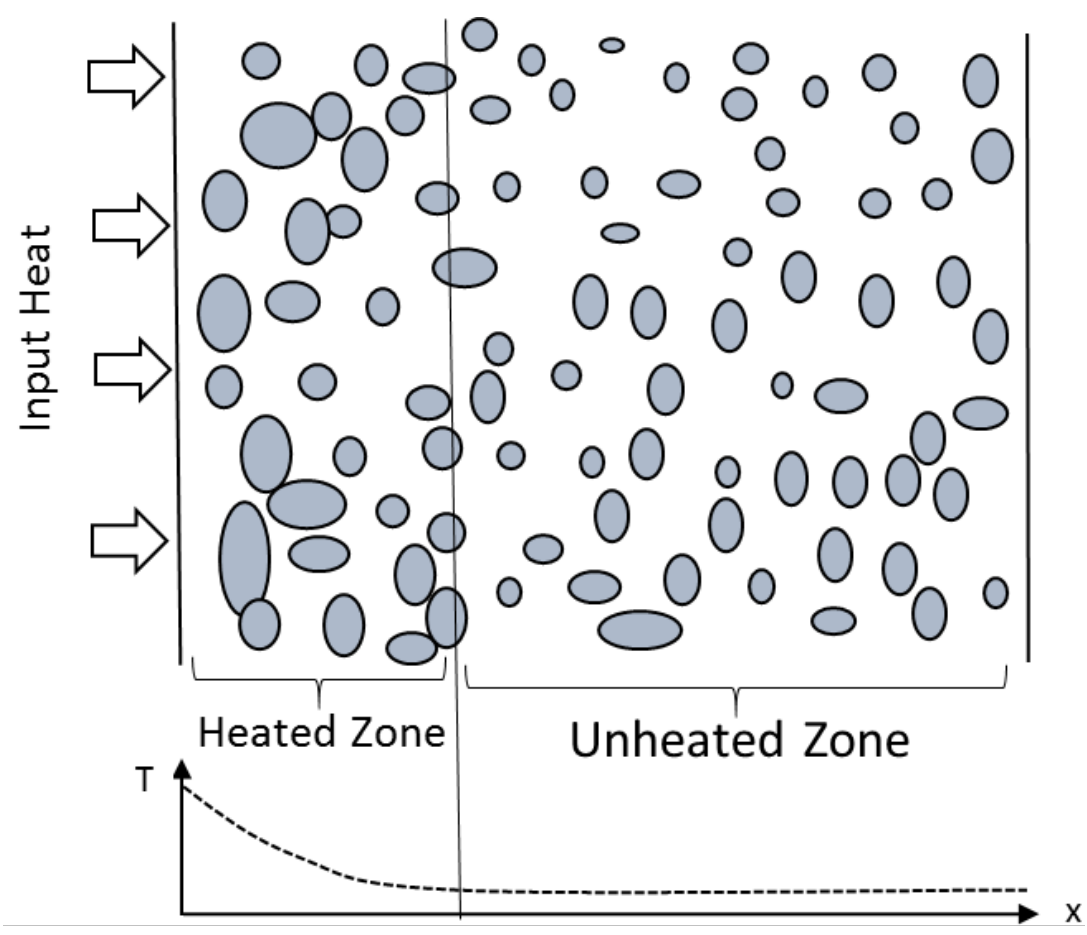

Fig. 2. Thermal response of brine-spongy ice to transient heat input 


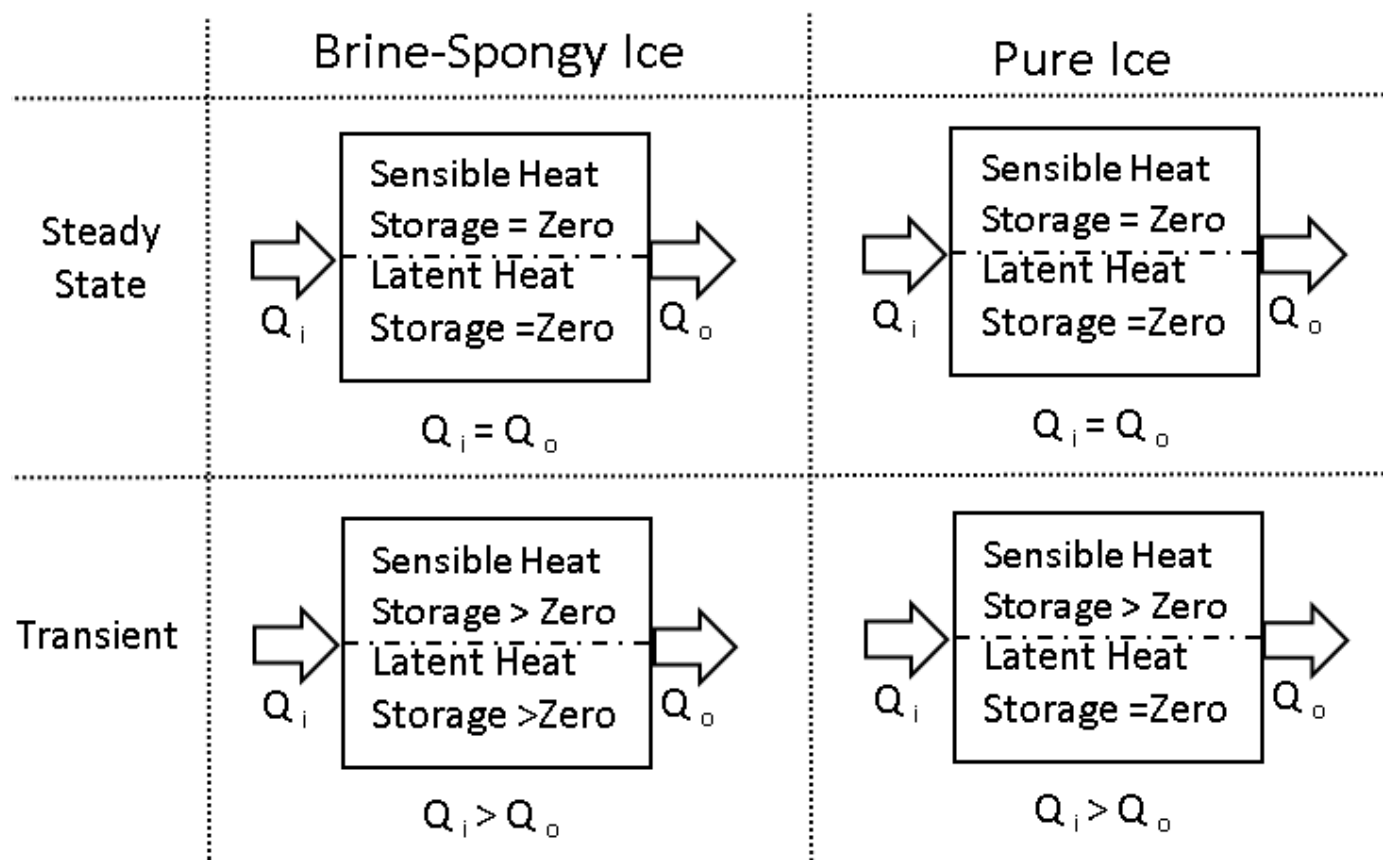

Fig. 3. Sensible and latent heat storage in brine-spongy and pure ice

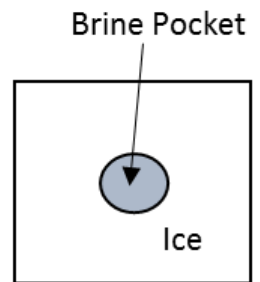

$\mathrm{t}=\mathrm{t}_{0}$

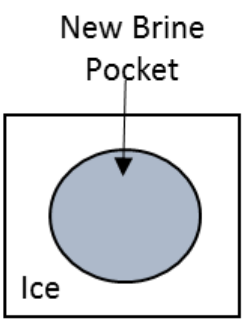

$t=t_{0}+\delta t$

Fig. 4. Small element of brine-spongy ice in a finite period of time 


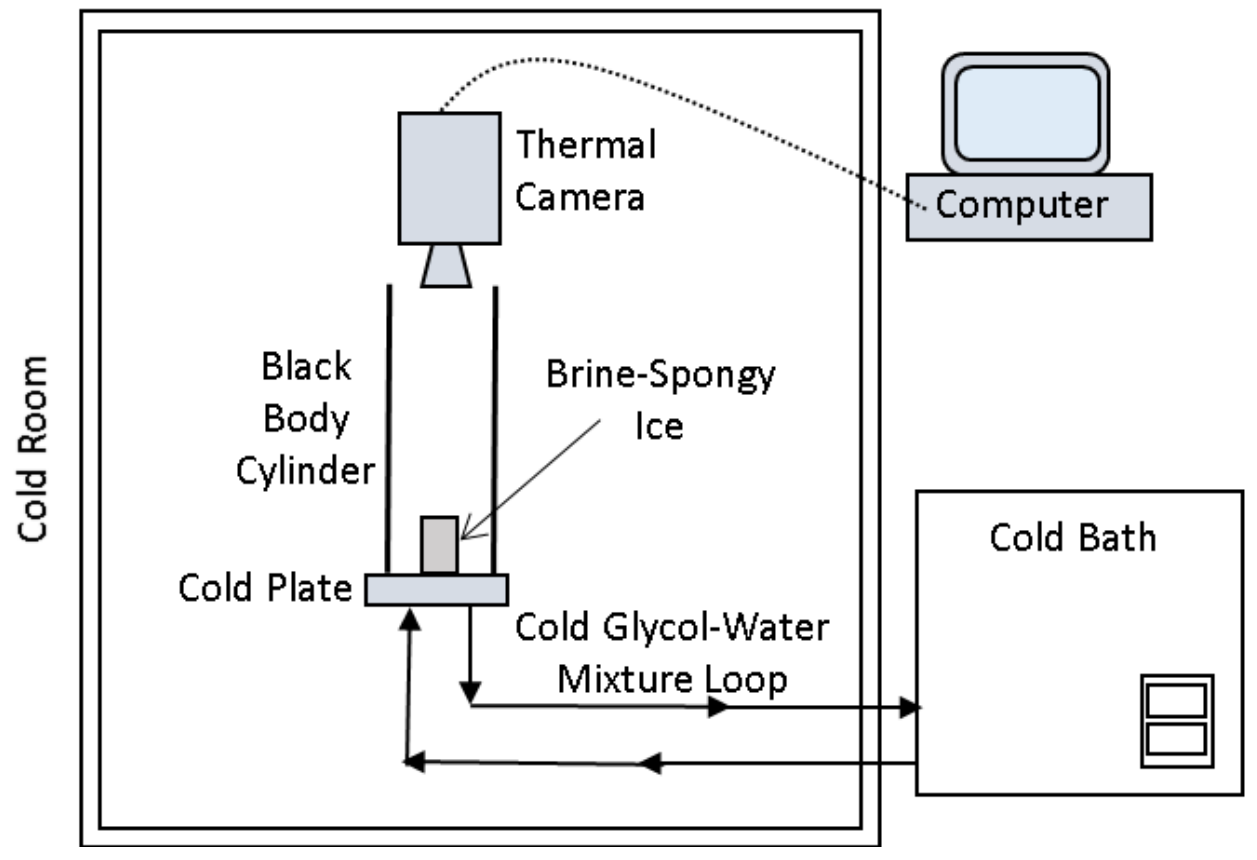

405

Fig. 5. Schematic of the experimental setup

406

407

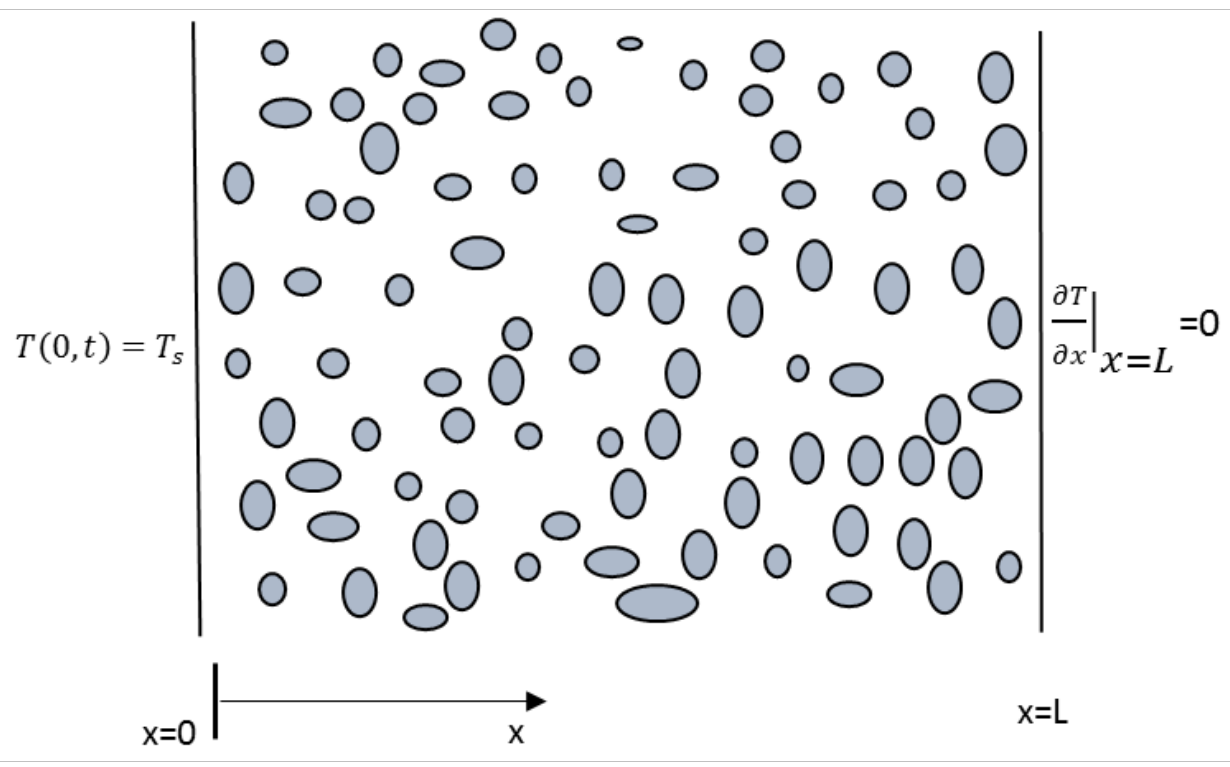

Fig. 6. Schematic of brine-spongy ice for the case study 


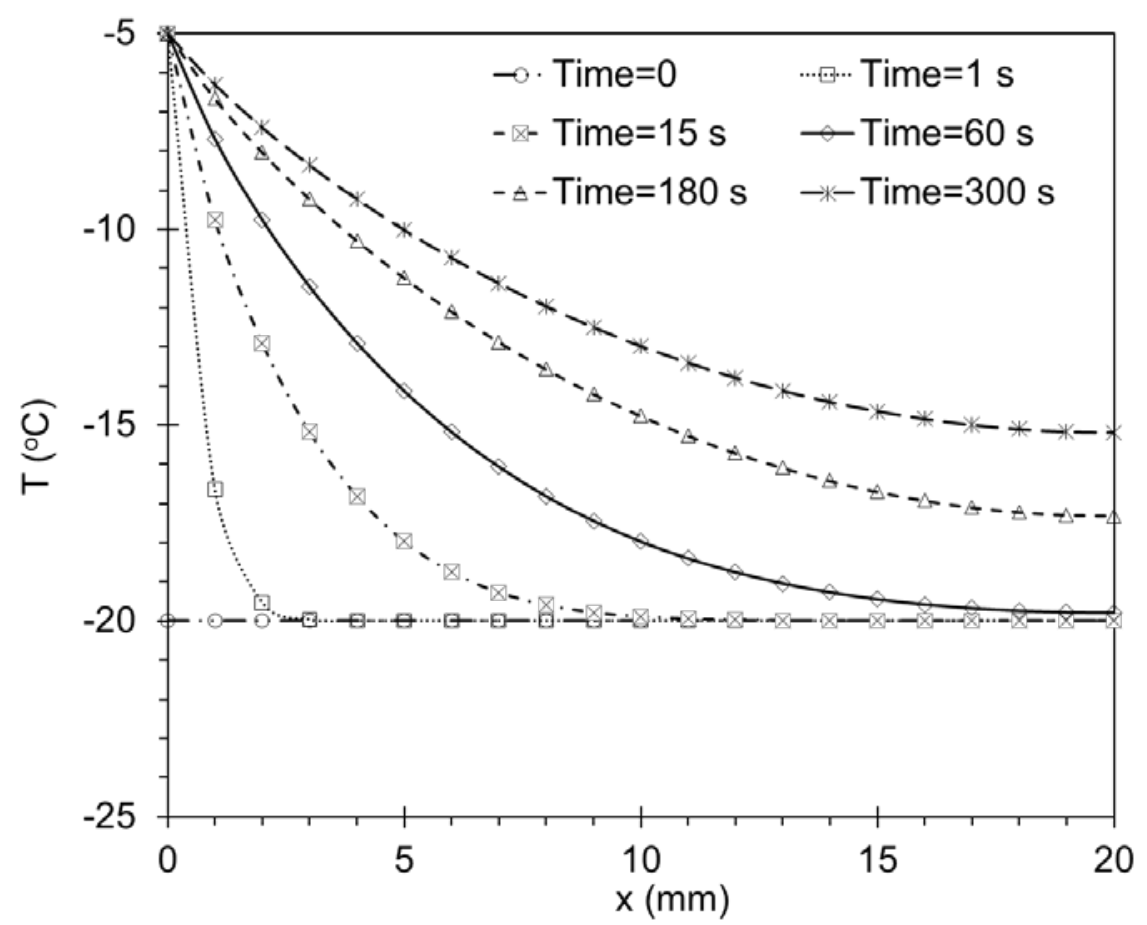

Fig. 7. Thermal response of brine-spongy ice

412

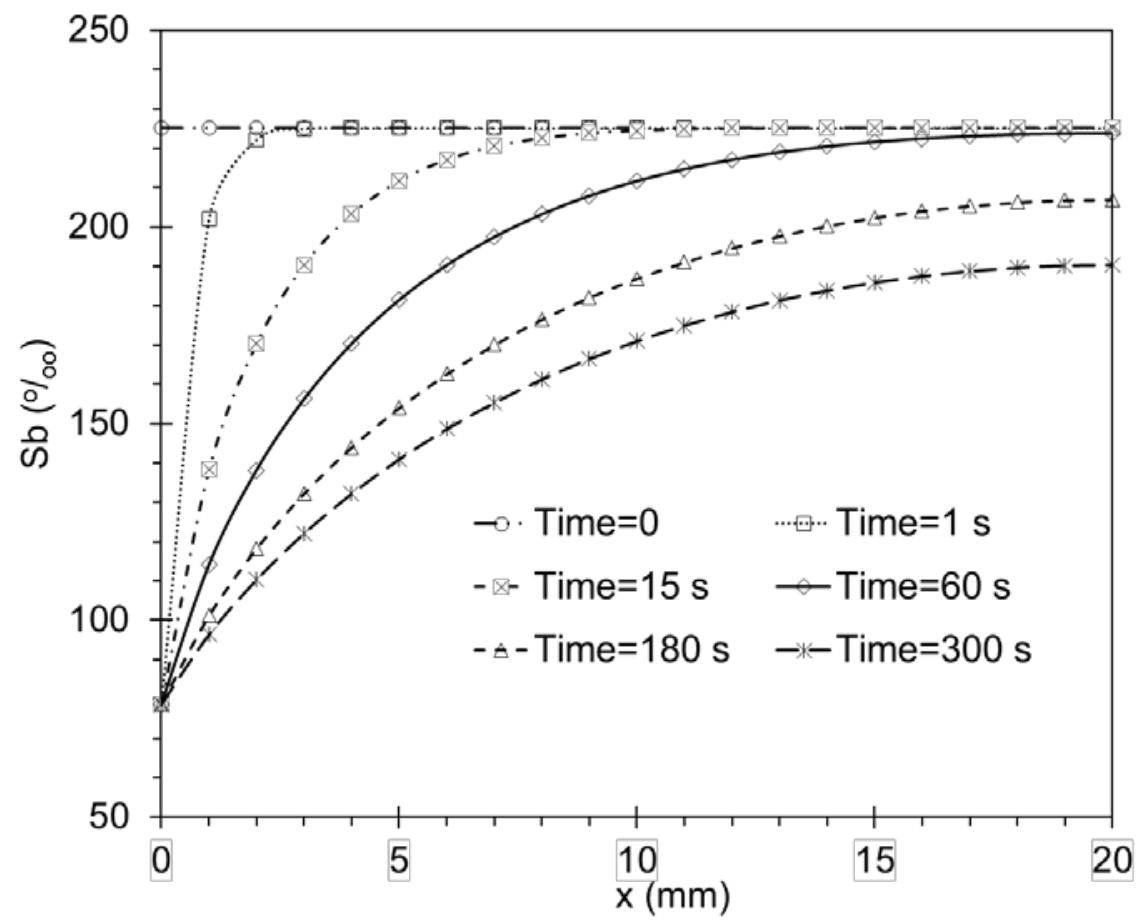

Fig. 8. Variation of salinity of brine pockets versus time 

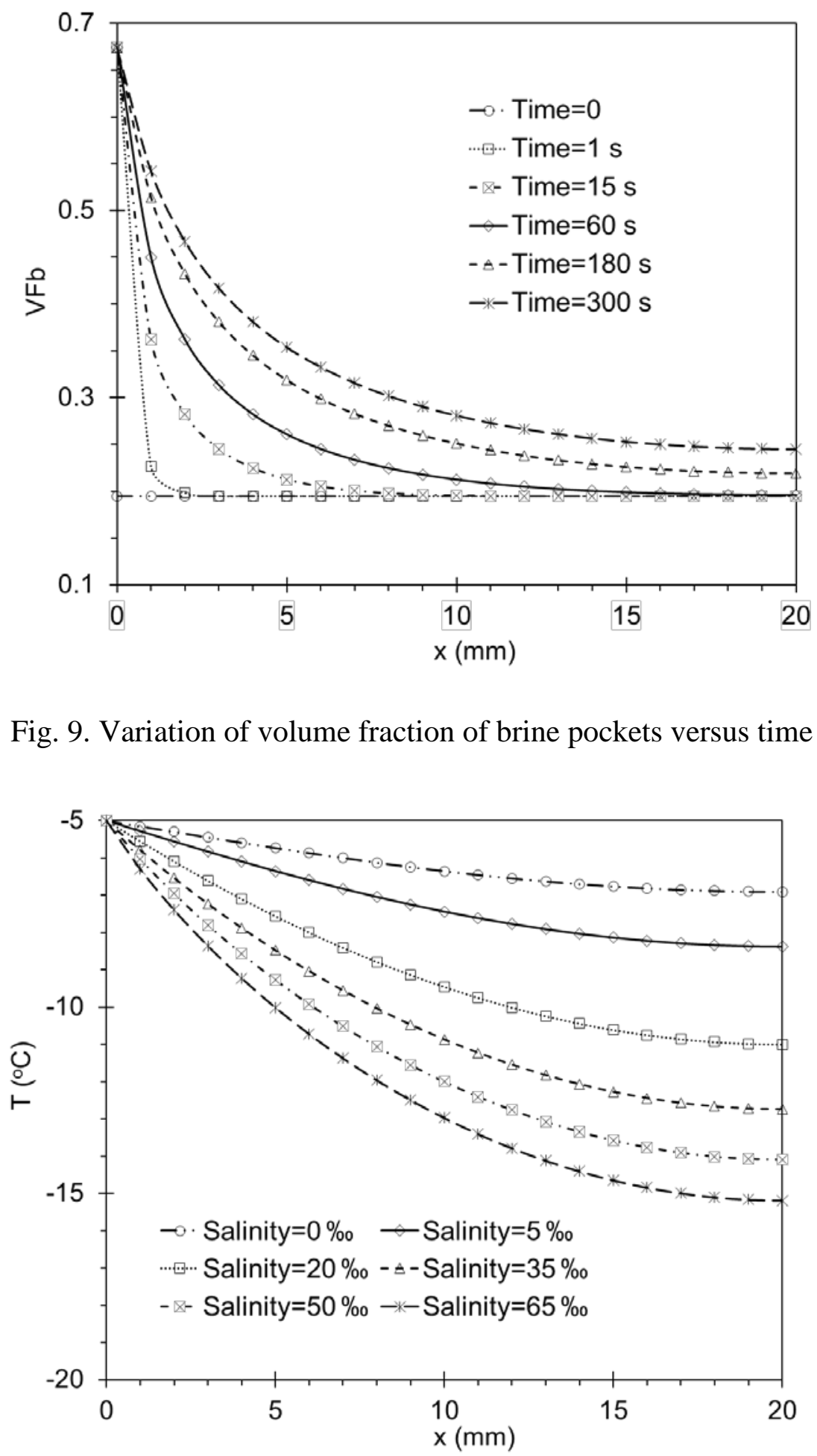

Fig. 10. Temperature variation of brine-spongy ice versus overall salinity 


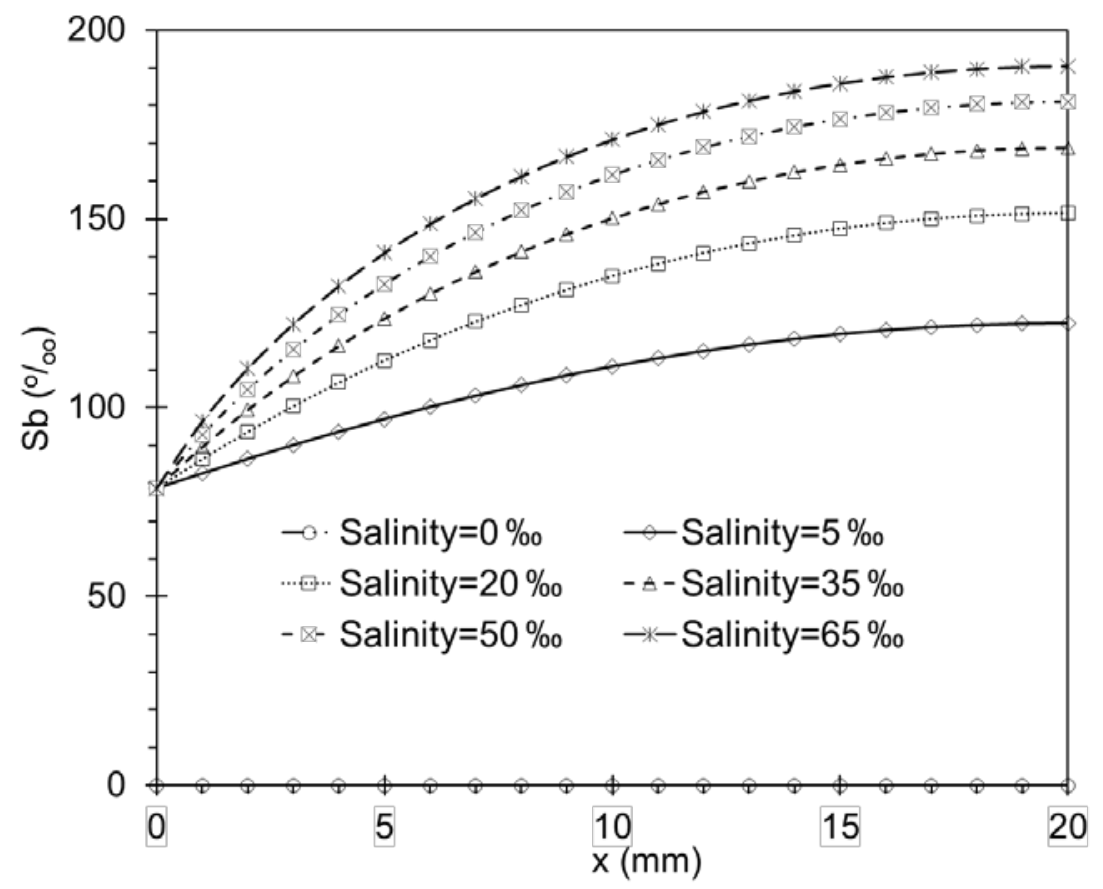

Fig. 11. Variation of salinity of brine pockets versus variation of overall salinity

423

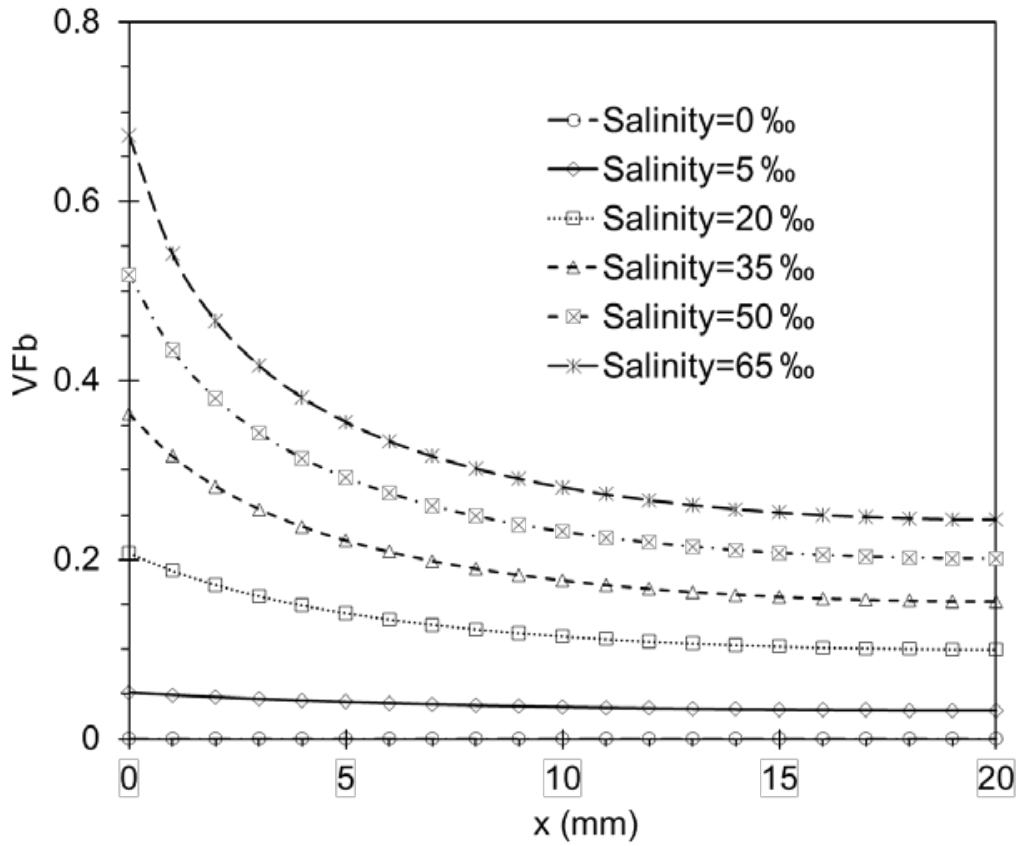

Fig. 12. Variation of brine volume fraction versus overall salinity 


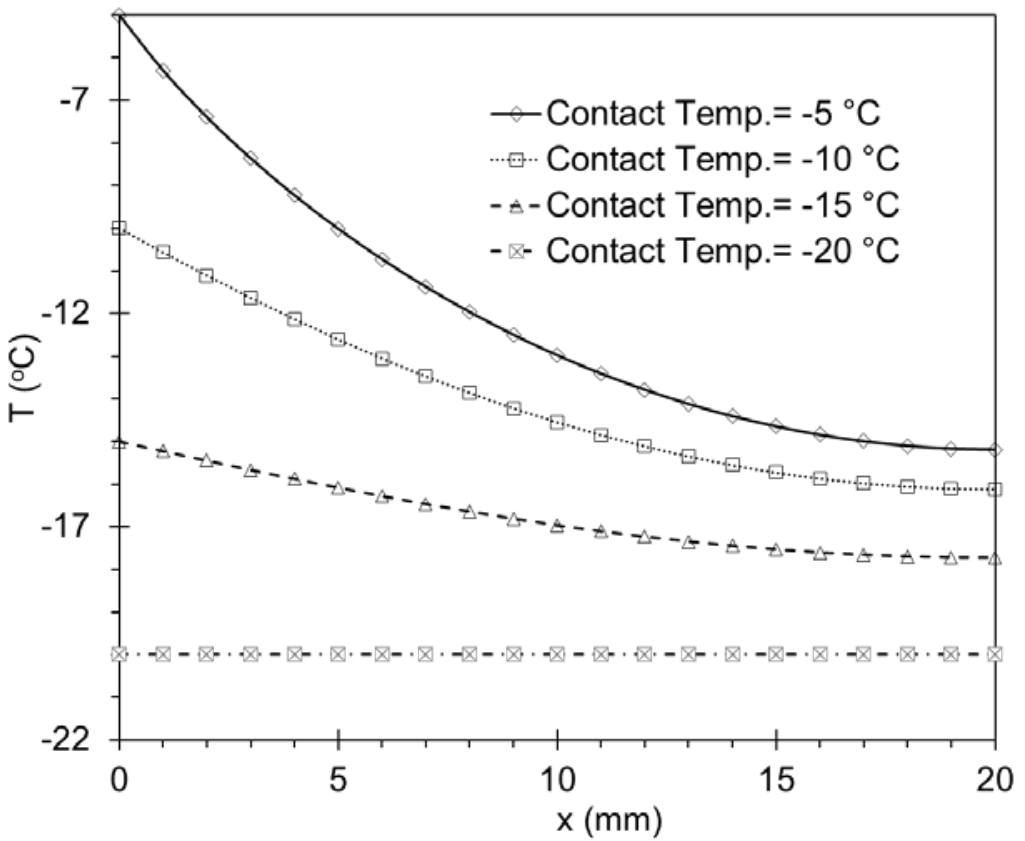

Fig. 13. Effects of contact temperatures on variation of temperature in brine-spongy ice

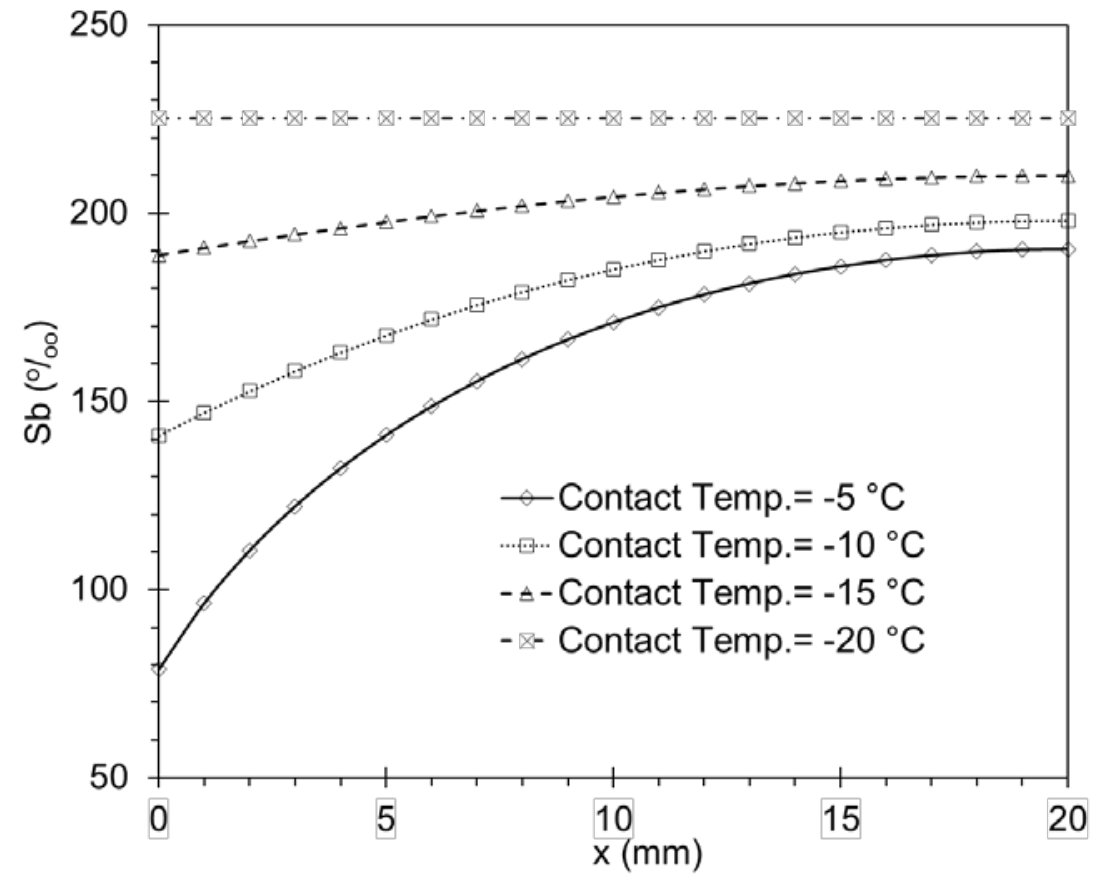

430

Fig. 14. Variation of salinity of brine pockets versus contact temperatures 

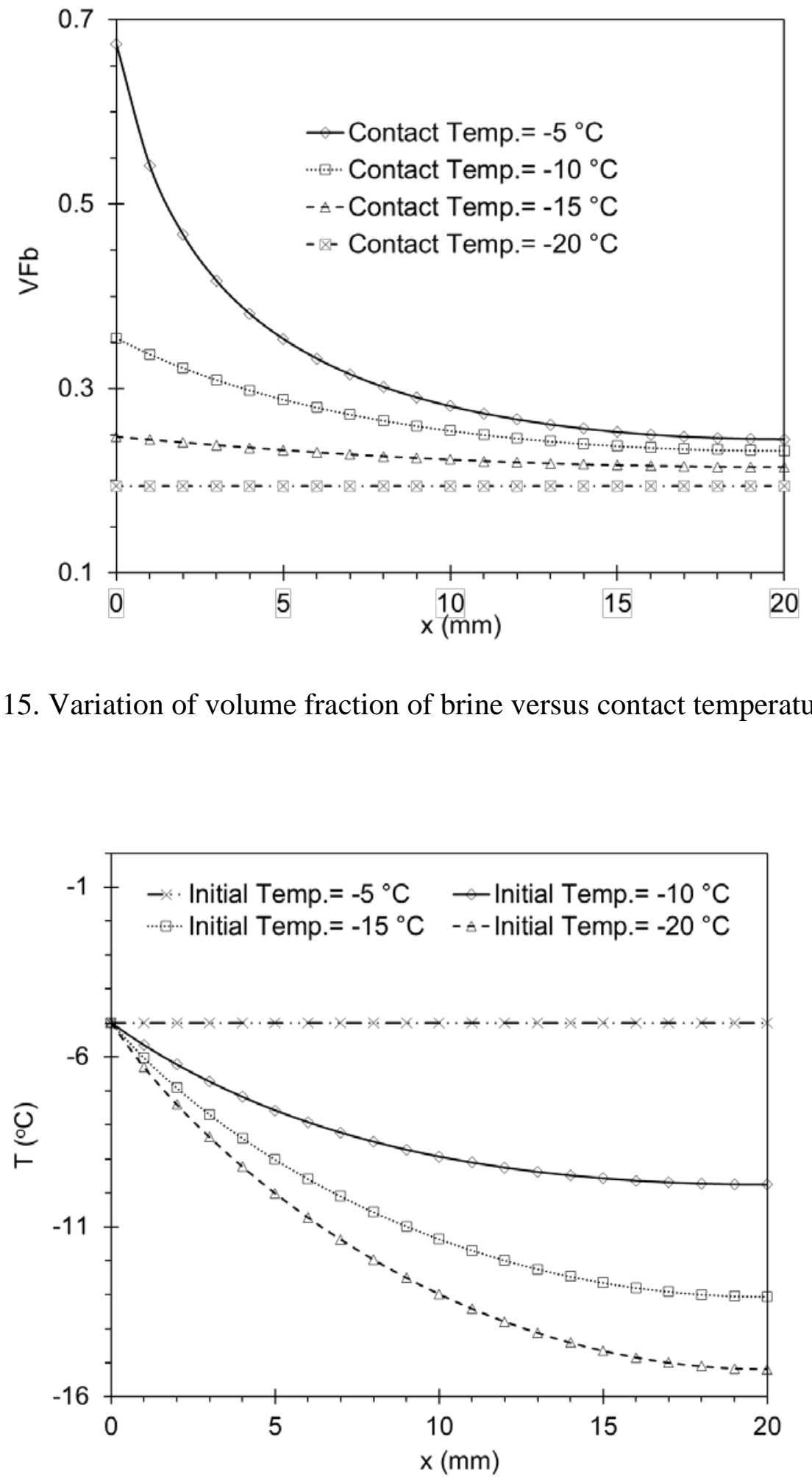

Fig. 16. Thermal response of brine-spongy ice in different initial temperatures 


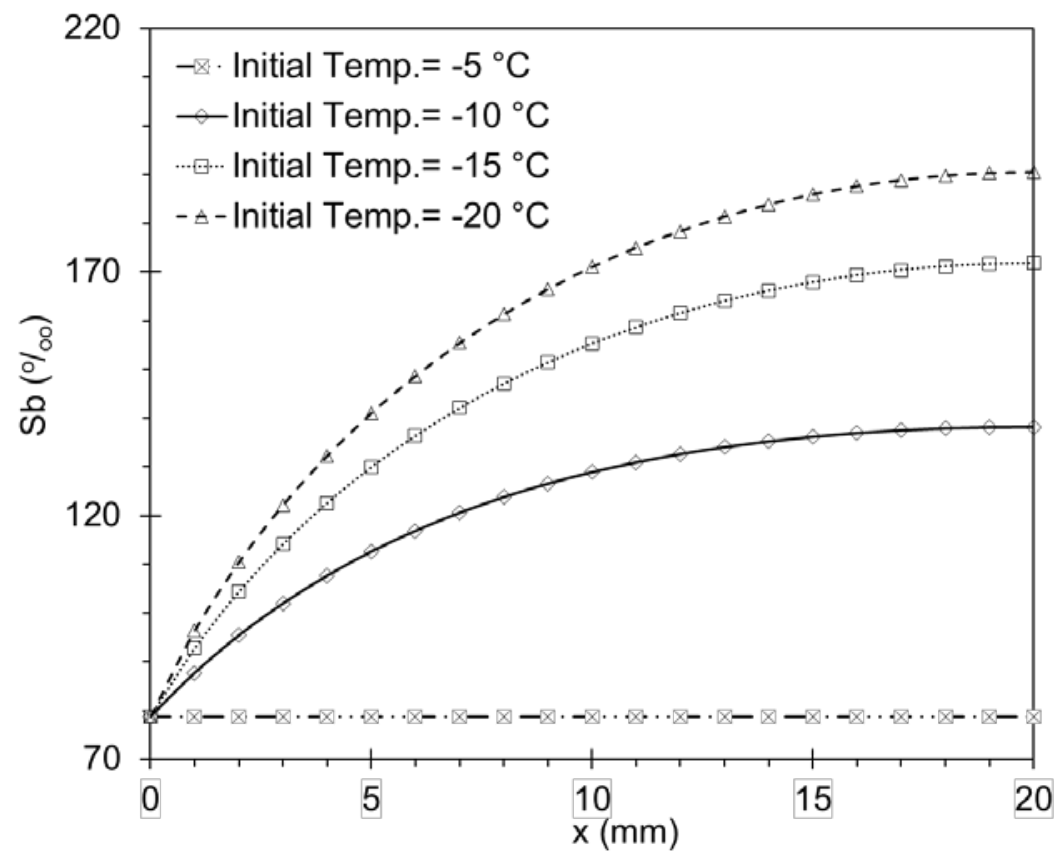

Fig. 17. Variation of salinity of brine pockets in various initial temperatures

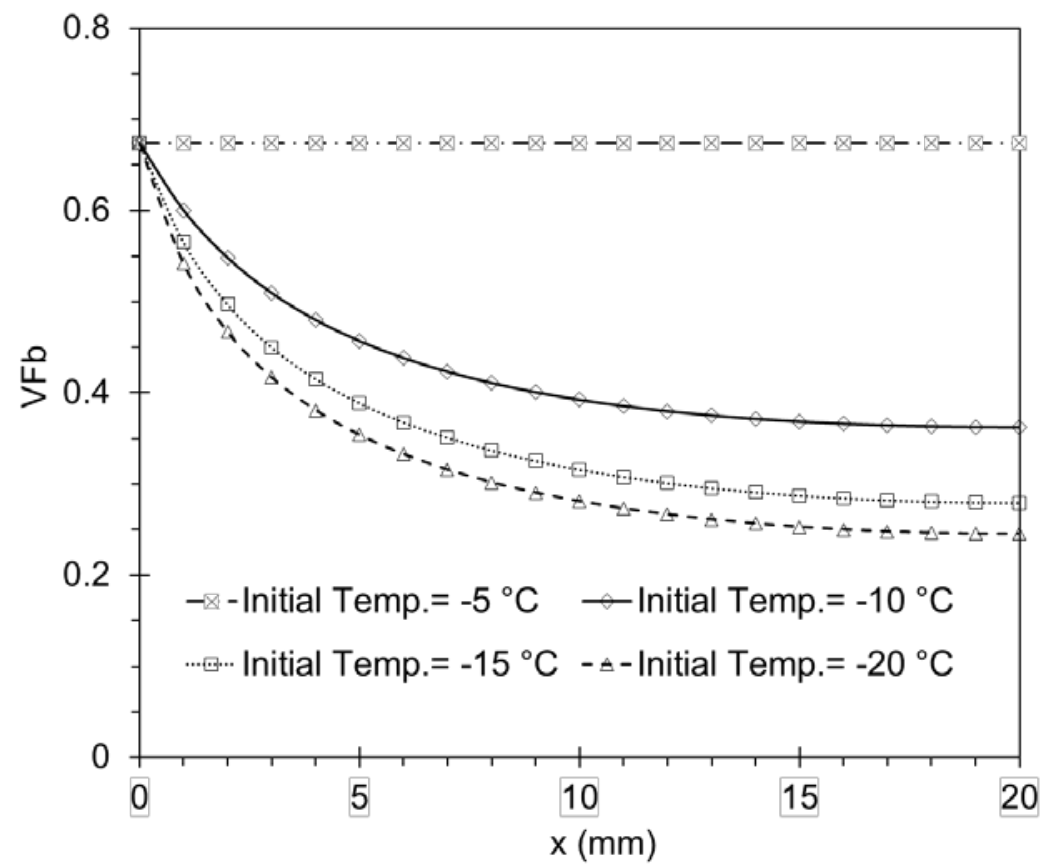

Fig. 18. Variation of volume fraction of brine in various initial temperatures 


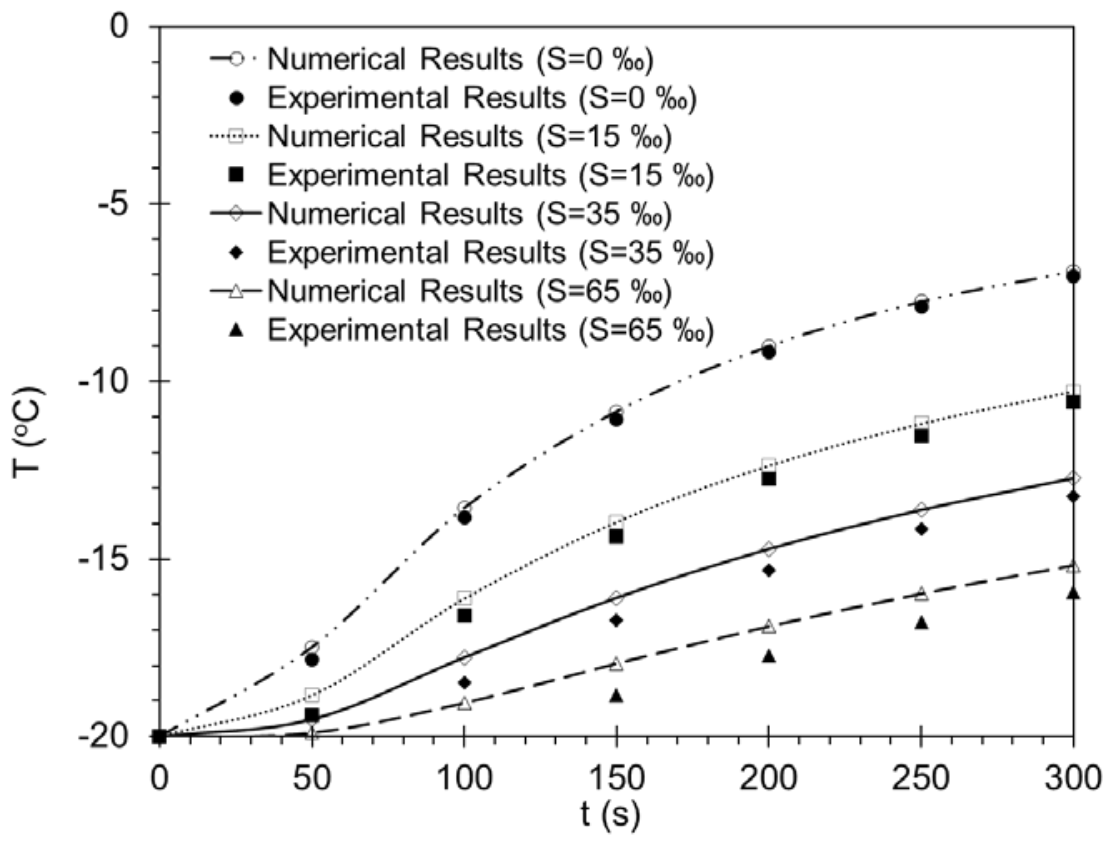

446 Fig. 19. Experimental and numerical results of temperature of brine-spongy ice block at various 
Table 1. Properties of ice, brine, and brine-spongy ice

\begin{tabular}{ll}
\hline Properties & Equations \\
\hline$S_{b}$ & $\alpha_{S b 1} T+\alpha_{S b 2} T^{2}+\alpha_{S b 3} T^{3}$ \\
$\rho_{b}$ & $\alpha_{\rho b 0}+\alpha_{\rho b 1} S_{b}$ \\
$L_{H b}$ & $\alpha_{L H b 0}+\alpha_{L H b 1} T+\alpha_{L H b 2} T^{2}$ \\
$C_{b}$ & $\alpha_{C b 0}+\alpha_{C b 1} T+\alpha_{C b 2} T^{2}+\alpha_{C b 3} T^{3}$ \\
$k_{b}$ & $\alpha_{k b 0}+\alpha_{k b 1} T+\alpha_{k b 2} T^{2}$ \\
$\rho_{i}$ & $\alpha_{\rho i 0}+\alpha_{\rho i 1} T$ \\
$C_{i}$ & $\alpha_{C i 0}+\alpha_{C i 1} T$ \\
$k_{i}$ & $\alpha_{k i 0}+\alpha_{k i 1} T$ \\
$k_{S}$ & $k_{b} V_{F b}+k_{i}\left(1-V_{F b}\right)$ \\
$\rho_{S}$ & $\rho_{b} V_{F b}+\rho_{i}\left(1-V_{F b}\right)$ \\
$C_{s}$ & $C_{b} V_{F b}+C_{i}\left(1-V_{F b}\right)$ \\
&
\end{tabular}


471

472

473

474

475

476

477

478

479

480

481

Table 2. Coefficients for equations of properties

\begin{tabular}{cllll}
\hline \multirow{2}{*}{ Coefficients } & \multicolumn{5}{c}{ Values } \\
\cline { 2 - 5 } & $(0)$ & $(1)$ & $(2)$ & $(3)$ \\
\hline$\alpha_{S b()}$ & 0 & -17.5730 & -0.381246 & $-3.28366 \times 10^{-3}$ \\
$\alpha_{L H b()}$ & -333400.326 & -4958.217 & -29.894 & 0 \\
$\alpha_{\rho b()}$ & 1000 & 0.8 & 0 & 0 \\
$\alpha_{C b()}$ & 4211.249 & 111.437 & 5.125 & $93.545 \times 10^{-3}$ \\
$\alpha_{k b()}$ & 0.5664 & $3.0822 \times 10^{-3}$ & $1.8388 \times 10^{-5}$ & 0 \\
$\alpha_{\rho i()}$ & 917 & -0.1403 & 0 & 0 \\
$\alpha_{C i()}$ & 2118.5199 & 7.8000 & 0 & 0 \\
$\alpha_{k i()}$ & 2.2399 & $-10.7517 \times 10^{-3}$ & 0 & 0 \\
\hline
\end{tabular}

